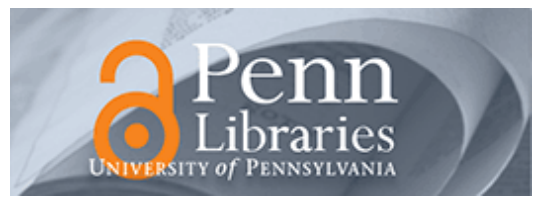

University of Pennsylvania

ScholarlyCommons

$1-20-1997$

\title{
Ferromagnetic Moment and Spin Rotation Transitions in Tetragonal Antiferromagnetic $\mathrm{Sr}_{2} \mathrm{Cu}_{3} \mathrm{O}_{4} \mathrm{Cl}_{2}$
}

Fangcheng Chou

Amnon Aharony

Robert J. Birgeneau

Ora Entin-Wohlman

Martin Greven

See next page for additional authors

Follow this and additional works at: https://repository.upenn.edu/physics_papers

Part of the Quantum Physics Commons

\section{Recommended Citation}

Chou, F., Aharony, A., Birgeneau, R. J., Entin-Wohlman, O., Greven, M., Harris, A., Kastner, M. A., Kim, Y., Kleinberg, D. S., Lee, Y., \& Zhu, Q. (1997). Ferromagnetic Moment and Spin Rotation Transitions in Tetragonal Antiferromagnetic $\mathrm{Sr}_{2} \mathrm{Cu}_{3} \mathrm{O}_{4} \mathrm{Cl}_{2}$. Physical Review Letters, 78 (3), 535-538. http://dx.doi.org/ 10.1103/PhysRevLett.78.535

This paper is posted at ScholarlyCommons. https://repository.upenn.edu/physics_papers/466

For more information, please contact repository@pobox.upenn.edu. 


\title{
Ferromagnetic Moment and Spin Rotation Transitions in Tetragonal Antiferromagnetic $\mathrm{Sr}_{2} \mathrm{Cu}_{3} \mathrm{O}_{4} \mathrm{Cl}_{2}$
}

\begin{abstract}
$\mathrm{Sr}_{2} \mathrm{Cu}_{3} \mathrm{O}_{4} \mathrm{Cl}_{2}$ is a variant of the lamellar copper oxides, containing an extra $\mathrm{Cu}^{2+}$ ion in the center of every second plaquette of the square $\mathrm{CuO}_{2}$ lattice. The two types of $\mathrm{Cu}$ form interpenetrating Heisenberg antiferromagnets, which order at 380 and $40 \mathrm{~K}$. Magnetization measurements yield a small spontaneous ferromagnetic moment below $380 \mathrm{~K}$ and two spin rotation transitions. The results are explained in detail by a pseudodipolar coupling between the two Cu lattices. A quantitative analysis of the data yields several previously unknown microscopic coupling constants, relevant to other lamellar, chain, and ladder copper oxides.
\end{abstract}

Disciplines

Physics | Quantum Physics

Author(s)

Fangcheng Chou, Amnon Aharony, Robert J. Birgeneau, Ora Entin-Wohlman, Martin Greven, A. Brooks Harris, Marc A. Kastner, Youngjune Kim, D. S. Kleinberg, Youngsu Lee, and Qing Zhu 


\title{
Ferromagnetic Moment and Spin Rotation Transitions in Tetragonal Antiferromagnetic $\mathrm{Sr}_{2} \mathrm{Cu}_{3} \mathrm{O}_{4} \mathrm{Cl}_{2}$
}

\author{
F. C. Chou, ${ }^{1}$ Amnon Aharony, ${ }^{1,2}$ R. J. Birgeneau, ${ }^{1}$ O. Entin-Wohlman, ${ }^{2}$ M. Greven, ${ }^{1}$ A. B. Harris,${ }^{3}$ \\ M. A. Kastner, ${ }^{1}$ Y. J. Kim, ${ }^{1}$ D. S. Kleinberg, ${ }^{1}$ Y. S. Lee, ${ }^{1}$ and Q. Zhu ${ }^{4}$ \\ ${ }^{1}$ Center of Materials Science and Engineering, Massachusetts Institute of Technology, Cambridge, Massachusetts 02139 \\ ${ }^{2}$ School of Physics and Astronomy, Raymond and Beverly Sackler Faculty of Exact Sciences, \\ Tel Aviv University, Tel Aviv 69978, Israel \\ ${ }^{3}$ Department of Physics, University of Pennsylvania, Philadelphia, Pennsylvania 19104 \\ ${ }^{4}$ Physics Department, Brookhaven National Laboratory, Upton, New York 11973
}

(Received 3 October 1996)

\begin{abstract}
$\mathrm{Sr}_{2} \mathrm{Cu}_{3} \mathrm{O}_{4} \mathrm{Cl}_{2}$ is a variant of the lamellar copper oxides, containing an extra $\mathrm{Cu}^{2+}$ ion in the center of every second plaquette of the square $\mathrm{CuO}_{2}$ lattice. The two types of $\mathrm{Cu}$ form interpenetrating Heisenberg antiferromagnets, which order at 380 and $40 \mathrm{~K}$. Magnetization measurements yield a small spontaneous ferromagnetic moment below $380 \mathrm{~K}$ and two spin rotation transitions. The results are explained in detail by a pseudodipolar coupling between the two $\mathrm{Cu}$ lattices. A quantitative analysis of the data yields several previously unknown microscopic coupling constants, relevant to other lamellar, chain, and ladder copper oxides. [S0031-9007(96)02201-6]
\end{abstract}

PACS numbers: 75.10.Jm, 75.25.+z, 75.30.Cr, 75.50.Ee

The magnetism of lamellar copper oxides has received much attention, because they both become high temperature superconductors when doped, and are also nearly ideal quantum antiferromagnets [1]. Therefore, these materials provide excellent testing grounds for theories of quantum magnetism. The fundamental building block of materials like $\mathrm{La}_{2} \mathrm{CuO}_{4}$ is the planar $\mathrm{CuO}_{2}$ layer consisting of a square lattice with $\mathrm{Cu}$ ions on the corners and $\mathrm{O}$ ions on the edges. The $\mathrm{Cu}^{2+}$ ions represent a $S=1 / 2$ square lattice quantum Heisenberg antiferromagnet (SLQHA), with a nearest neighbor $(\mathrm{NN})$ leading isotropic $\mathrm{O}$-mediated superexchange energy $J_{0} \approx 130 \mathrm{meV}$. These materials have nonzero Néel temperatures due to small interplanar coupling and spin anisotropies [1-3].

Recently, attention has focused on "234" variants of the cuprates, $\mathrm{Sr}_{2} \mathrm{Cu}_{3} \mathrm{O}_{4} \mathrm{Cl}_{2}$ and $\mathrm{Ba}_{2} \mathrm{Cu}_{3} \mathrm{O}_{4} \mathrm{Cl}_{2}$ [4-6]. These materials have an additional $\mathrm{Cu}$ ion (denoted by $\mathrm{Cu}$ II) at the center of every second plaquette of the $\mathrm{CuO}_{2}$ lattice (made of $\mathrm{Cu}$ I's), creating two interpenetrating square lattices of $\mathrm{Cu}$ ions (see Fig. 1) [7]. Similarly to other lamellar cuprates, neutron scattering experiments find $\mathrm{Cu}$ I antiferromagnetic (AFM) order below $T_{N, \mathrm{I}} \approx 380 \mathrm{~K}$. In addition, the $\mathrm{Cu}$ II's exhibit AFM order below $T_{N, \text { II }} \approx 40 \mathrm{~K}$ $[4,6,8]$. The detailed nature of the $\mathrm{Cu}$ II-Cu II and the $\mathrm{Cu}$ I$\mathrm{Cu}$ II interactions has not yet been identified. Concerning the former, we provide evidence that, like the $\mathrm{Cu}$ I's, the $\mathrm{Cu}$ II's represent a SLQHA. The $\mathrm{Cu}$ I-Cu II interactions are expected to be quite delicate; since each $\mathrm{Cu}$ II is surrounded by four $\mathrm{Cu}$ I's, an isotropic Heisenberg interaction would yield frustration, due to a vanishing mean field at the $\mathrm{Cu}$ II sites. The present Letter presents information on both the isotropic and the crucial anisotropic terms, as well as on quantum fourfold terms.

The $\mathrm{Cu}$ I-O-Cu II and $\mathrm{Cu}$ II-O-O-Cu II geometry in 234 is, respectively, the same as that for the $\mathrm{NN}$ and next
$\mathrm{NN}(\mathrm{NNN}) \mathrm{Cu}-\mathrm{Cu}$ pairs in the spin chains which arise, e.g., in $\mathrm{Sr}_{14} \mathrm{Cu}_{24} \mathrm{O}_{41}$ [9]. Our $\mathrm{Cu}$ I-O-Cu II geometry is also the same as the interladder one in $\mathrm{Sr}_{14} \mathrm{Cu}_{24} \mathrm{O}_{41}$ and in many ladder cuprates, like $\mathrm{Sr}_{n-1} \mathrm{Cu}_{n+1} \mathrm{O}_{2 n}$ [10]. Thus,

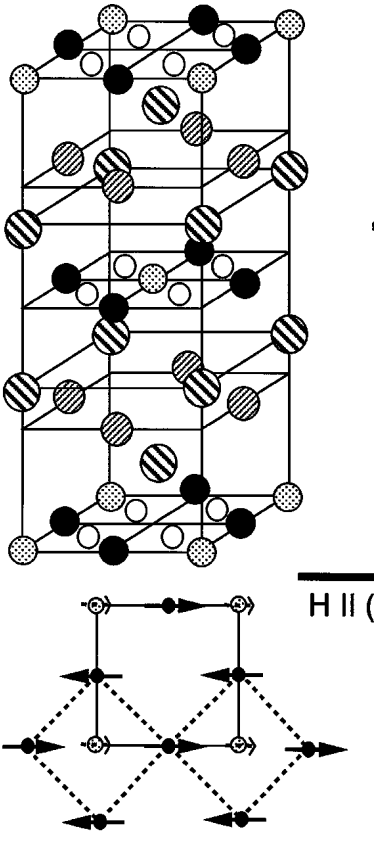

(b)

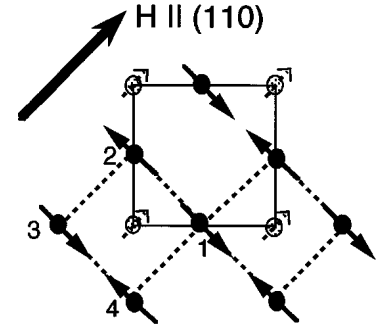

(a)

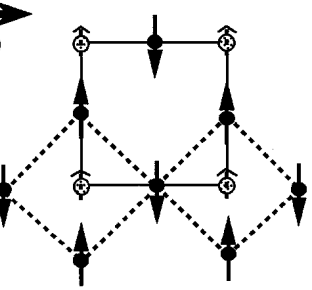

(c)
FIG. 1. Structure of $\mathrm{Sr}_{2} \mathrm{Cu}_{3} \mathrm{O}_{4} \mathrm{Cl}_{2}$ and of the $\mathrm{Cu}_{3} \mathrm{O}_{4}$ layer, including spin configurations for (a) $\mathbf{H} \|$ (110), (b) $\mathbf{H} \|$ (100) and $H_{c 1}<H<H_{c 2}$, and (c) $\mathbf{H} \|(100)$ and $H_{c 2} \ll H$. The figure shows only the part of $\mathbf{M}_{\mathrm{II}}$ induced by the internal pseudodipolar field $-4 J_{\mathrm{pd}} \hat{\Gamma} \mathbf{M}_{\mathrm{I}}^{\dagger}$. A nonzero $H$ induces an additional small canting of the $\mathrm{Cu}$ I moments in cases (a) and (c), and a large component of $\mathbf{M}_{\mathrm{II}}$ along $\mathbf{H}$ in case (c) (not shown). 
our measured coupling constants in 234 represent estimates for their hitherto unmeasured counterparts in many chain, ladder, and planar cuprates.

Perhaps the most peculiar feature of 234 is a small spontaneous ferromagnetic (FM) moment, $M_{S} \sim 10^{-3} \mu_{B}$, which appears at $T_{N, \mathrm{I}} . \quad M_{S}$ has been attributed [4-6] to the Dzyaloshinsky-Moriya (DM) antisymmetric exchange. Although this mechanism is effective in orthorhombic $\mathrm{La}_{2} \mathrm{CuO}_{4}[11]$, symmetry forbids it in the perfectly tetragonal structure of 234: the DM interactions from individual bonds sum up to zero. Thus, the origin of $M_{S}$ has been a mystery. Here we show that in tetragonal symmetry $M_{S}$ must result from anisotropic pseudodipolar $\mathrm{Cu}$ I-Cu II interactions. Similar interactions have been predicted between $\mathrm{NN}$ planes in $\mathrm{Sr}_{2} \mathrm{CuO}_{2} \mathrm{Cl}_{2}$ or $\mathrm{Nd}_{2} \mathrm{CuO}_{4}$ [12], but have not yet been measured. We report measurements of the field dependence of the magnetization of $\mathrm{Sr}_{2} \mathrm{Cu}_{3} \mathrm{O}_{4} \mathrm{Cl}_{2}$, $M(H)$, for different directions of the field $\mathbf{H}$, which exhibit a rich behavior [including two spin rotation transitions for H || (100)], providing detailed quantitative evidence for this theoretical description.

We have focused on $\mathrm{Sr}_{2} \mathrm{Cu}_{3} \mathrm{O}_{4} \mathrm{Cl}_{2}$, for which it is possible to grow large single crystals by slow cooling from a melt containing $\mathrm{CuO}$ as flux. Small crystals, $\sim 6 \mathrm{~mm} \times 7 \mathrm{~mm} \times 0.5 \mathrm{~mm}$, with the $c$ axis (normal to the $\mathrm{Cu}_{3} \mathrm{O}_{4}$ layer) perpendicular to the large face are used for measurements of $M(H)$ with a quantum design SQUID magnetometer at $H<5.5 \mathrm{~T}$. High-resolution synchrotron $\mathrm{x}$-ray powder diffraction measurements show that the crystal remains tetragonal (space group $I 4 / \mathrm{mmm}$ ) for temperatures $15<T<550 \mathrm{~K}$, with lattice contants $a=$ $5.457 \AA$ and $c=12.52 \AA$ for $T<50 \mathrm{~K}$. (The effects of the lower magnetic symmetry are negligible.)

Our measured $M(H)$ becomes simplest for $\mathbf{H} \|(110)$ : the susceptibility $\chi \equiv d M / d H$ is independent of $H$ for all $H>0.1 \mathrm{~T}$, and the data in this range are fitted by $M(H)=$ $M_{S}^{(110)}(T)+\chi^{(110)}(T) H$. For $H<0.1 \mathrm{~T}$, the system exhibits interesting domain effects [13]. The spontaneous moment $M_{S}^{(110)}(T)$ is plotted in Fig. 2. The full line in that figure represents a fit near $T_{N, \mathrm{I}}$ to $\left(T_{N, \mathrm{I}}-T\right)^{\beta}$, with $\beta=$

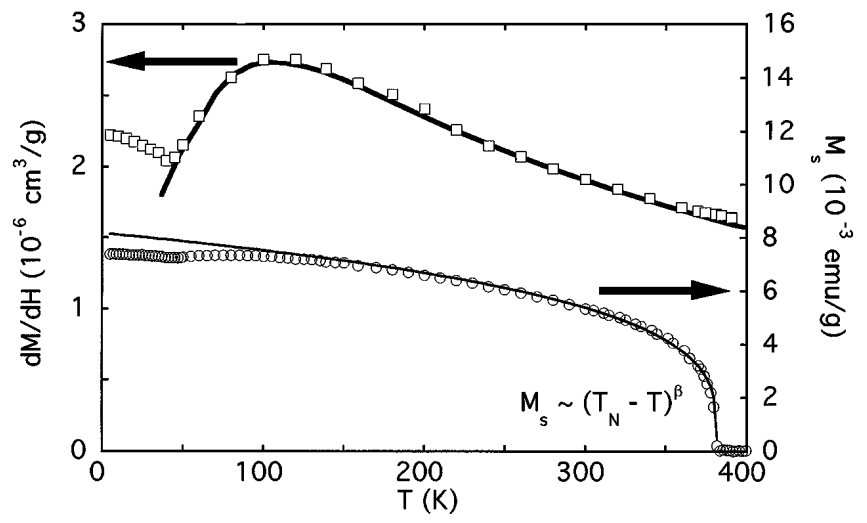

FIG. 2. Saturated FM moment $M_{S}^{(110)}$ and susceptibility $\chi^{(110)}$. The full lines represent $M_{S} \sim\left(T_{N, \mathrm{I}}-T\right)^{\beta}$ and a simulation of $\chi_{\text {II }}$ for the $S=1 / 2$ SLQHA (see text).
$0.27 \pm 0.03$ and $T_{N, \mathrm{I}}=(382 \pm 2) \mathrm{K} . \quad \beta$ agrees within the errors with those found for the staggered moment $M_{\mathrm{I}}^{\dagger}$ of the $\mathrm{Cu}$ I spins [6,8] and for $M^{\dagger}$ in $\mathrm{Sr}_{2} \mathrm{CuO}_{2} \mathrm{Cl}_{2}$ [1] and $\mathrm{La}_{2} \mathrm{CuO}_{4}$ [14]. The proportionality $M_{S} \sim M_{\mathrm{I}}^{\dagger}$ indicates that the $\mathrm{Cu}$ II spins see an effective uniform field proportional to $M_{\mathrm{I}}^{\dagger}$. Such a field would arise from a bilinear coupling between $M_{S}$ and $M_{\mathrm{I}}^{\dagger}$, but this is rather unusual for tetragonal systems.

Figure 2 also shows $\chi^{(110)}(T)$. In principle, $\chi=$ $\chi_{\mathrm{II}}+2 \chi_{\mathrm{I}, \perp}+\chi_{d}+\chi_{\mathrm{VV}}$, with the diamagnetic core susceptibility $\chi_{d} \sim-3.3 \times 10^{-7} \mathrm{~cm}^{3} / \mathrm{g}$ [15], the small contribution of the $\mathrm{Cu}$ I's, $2 \chi_{\mathrm{I}, \perp} \sim 4 \times 10^{-7} \mathrm{~cm}^{3} / \mathrm{g}$ (see below), and the Van Vleck susceptibility $\chi_{\mathrm{Vv}}(\sim 0.5 \times$ $10^{-7} \mathrm{~cm}^{3} / \mathrm{g}$, for $\mathrm{La}_{2} \mathrm{CuO}_{4}$ [16]). However, fortuitously the last 3 terms add up to zero within the errors, and we have $\chi \approx \chi_{\mathrm{II}}$. The solid heavy curve in Fig. 2 represents results for $\chi_{\text {II }}$ from Monte Carlo simulations of the $S=1 / 2$ SLQHA [17], with the single isotropic exchange parameter $J_{\mathrm{II}}=(10 \pm 1) \mathrm{meV}$ [18]. The detailed quantitative agreement of the simulations with the measured $\chi_{\text {II }}$ shows that the $\mathrm{Cu}$ II system is a new example of a $S=1 / 2$ SLQHA. The neutron results confirm this in detail, and take advantage of the small $J_{I I}$ to measure the first spin wave spectra of a SLQHA [8]. The actual ordering at $T_{N, \text { II }}$ results mainly from an effective Ising anisotropy, caused by the presence of the FM moment on $\mathrm{Cu}$ II. Indeed, the neutron experiments for $M_{\mathrm{II}}^{\dagger}$ below $40 \mathrm{~K}$ are well fitted with the two dimensional Ising exponent $\beta=1 / 8$ [8]. The cusp in $\chi$ at $40 \mathrm{~K}$ is also typical of an AFM ordering, giving more support for our identification of $\chi$ with $\chi_{\mathrm{II}}$.

When $\mathbf{H}$ is not along (110), $\chi$ becomes $H$ dependent, and $M$ becomes smaller than its value along (110), implying competing interactions. We thus identify (110) as the easy axis. For large $H$, the staggered moment of the $\mathrm{Cu}$ I's becomes perpendicular to $\mathbf{H}$, gaining from the larger perpendicular susceptibility $\chi_{\mathrm{I}, \perp}$. Therefore, $\chi$ approaches the same limit $\chi^{(110)}$, for all the directions of $\mathbf{H}$. To emphasize the deviations from these parallel asymptotes, we plot $m(H, \alpha)=\left(M-\chi^{(110)} H\right) / M_{S}^{(110)}$, when $\mathbf{H} \equiv$ $H(\cos \alpha, \sin \alpha, 0)$ (Fig. 3). At $T=200 \mathrm{~K}$ and $\alpha=0, \chi$ becomes $H$ independent between two critical fields, $H_{c 1}=$ $0.3 \mathrm{~T}$ and $H_{c 2}=1.7 \mathrm{~T}$, indicated by arrows in Fig. 3, implying that $M(H)=M_{S}^{\mathrm{b}}+\chi^{\mathrm{b}} H$, with $\chi^{\mathrm{b}}<\chi^{(110)}$ and $M_{S}^{\mathrm{b}}<M_{S}^{(110)}$. $\chi$ does vary with $H$ outside of this intermediate region, with discontinuities at $H_{c 1}$ and $H_{c 2}$. As we show below, the variation of $\chi$ with $H$ reflects a rotation of $\mathbf{M}_{\mathrm{I}}^{\dagger}$ between the structures shown in Figs. 1(a)-1(c). We observe no such sharp transitions at $\alpha \neq 0$, or at lower $T$.

We now introduce our theoretical model. Since we ignore the $\mathrm{Cu}$ II- $\mathrm{Cu}$ II interactions [13], the following results apply only for $T_{N, \text { II }}<T<T_{N, \text { I }}$. Consider a Cu I-Cu II bond along $\hat{x} \equiv(100)$ (Fig. 1). Since there exist two mirror planes which contain this bond, one perpendicular to the tetragonal $z$ axis and the other containing that axis, the most general form allowed for the symmetric $\mathrm{Cu}$ I-Cu II 


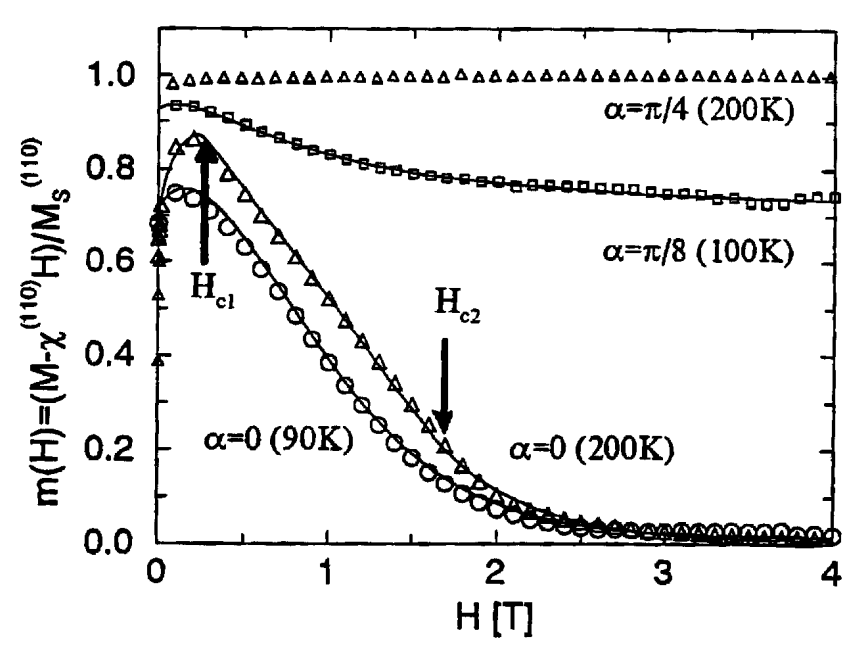

FIG. 3. Theoretical (full lines) and measured values of $m(H, \alpha)$.

interaction must be of the form

$$
\mathcal{H}_{\mathrm{I}-\mathrm{II}}=J^{\|} S_{\mathrm{I}}^{\|} S_{\mathrm{II}}^{\|}+J^{\perp} S_{\mathrm{I}}^{\perp} S_{\mathrm{II}}^{\perp}+J^{z} S_{\mathrm{I}}^{z} S_{\mathrm{II}}^{z},
$$

where $\|$ and $\perp$ denote parallel and perpendicular to the $\mathrm{Cu}$ I-Cu II bond [12]. Denoting the $4 \mathrm{Cu}$ I's adjacent to a $\mathrm{Cu}$ II by $1,2,3$, and 4 for the $\hat{x}, \hat{y},-\hat{x}$, and $-\hat{y}$ directions, and restricting all the spins to the $X Y$ plane (as found experimentally), the sum over these four bonds yields

$$
\mathcal{H}_{\mathrm{I}-\mathrm{II}}=4 \mathbf{M}_{\mathrm{II}} \cdot\left(J_{\mathrm{av}} \mathbf{M}_{\mathrm{I}}+J_{\mathrm{pd}} \hat{\Gamma} \mathbf{M}_{\mathrm{I}}^{\dagger}\right),
$$

where $2 J_{\mathrm{av}}=\left(J^{\|}+J^{\perp}\right), \quad 2 J_{\mathrm{pd}}=\left(J^{\|}-J^{\perp}\right), \quad \mathbf{M}_{\mathrm{II}}=$ $\mathbf{S}_{\mathrm{II}}$, while $\mathbf{M}_{\mathrm{I}}=\sum_{\mathrm{I}=1}^{4} \mathbf{S}_{\mathrm{I}, i} / 4$ and $\mathbf{M}_{\mathrm{I}}^{\dagger}=\left(\mathbf{S}_{\mathrm{I}, 1}-\mathbf{S}_{\mathrm{I}, 2}+\right.$ $\left.\mathbf{S}_{\mathrm{I}, 3}-\mathbf{S}_{\mathrm{I}, 4}\right) / 4$ represent the FM and AFM Cu I moments [18]. Here, $\hat{\Gamma} \equiv \sigma_{z}$ is the $2 \times 2$ Pauli matrix: $\hat{\Gamma}(x, y) \equiv(x,-y)$. In addition to the isotropic average $J_{\mathrm{av}}, J_{\mathrm{pd}}$ represents an anisotropic "pseudodipolar" net interaction, which yields a bilinear coupling between $\mathbf{M}_{\mathrm{I}}^{\dagger}$ and $\mathbf{M}_{\mathrm{II}}$. As soon as $\mathbf{M}_{\mathrm{I}}^{\dagger}$ orders, it generates a net field $-4 J_{\mathrm{pd}} \hat{\Gamma} \mathbf{M}_{\mathrm{I}}^{\dagger}$ on the $\mathrm{Cu}$ II in the center of each plaquette. Since the Cu II's occupy only every second plaquette, they are all surrounded by exactly the same configuration of $\mathrm{Cu}$ I moments (both in each plane and in adjacent planes) [8]. Therefore they all see the same local field and have the same FM moment [Figs. 1(a)-1(c)] [19].

If $\mathbf{M}_{\mathrm{I}}^{\dagger} \equiv M_{\mathrm{I}}^{\dagger}(\cos \theta, \sin \theta)$, the second term in Eq. (2) is minimized when $\mathbf{M}_{\mathrm{II}} \| \hat{\Gamma} \mathbf{M}_{\mathrm{I}}^{\dagger}=M_{\mathrm{I}}^{\dagger}(\cos \theta,-\sin \theta)$, and the energy of this term is the same for all $\theta$. In particular, the cases $\theta=-\pi / 4,0$ and $-\pi / 2$, shown in Figs. 1(a)$1(\mathrm{c})$, respectively, have the same energy, which is the minimum of this term for $J_{\mathrm{pd}}<0$. However, once $\mathbf{M}_{\mathrm{II}} \neq$ 0 (due to the second term), the first term generates a small FM moment $\mathbf{M}_{\mathrm{I}} \| \mathbf{M}_{\mathrm{II}}$. Since $\chi_{\mathrm{I}, \perp}>\chi_{\mathrm{I}, \|}(\|$ and $\perp$ to $\mathbf{M}_{\mathrm{I}}^{\dagger}$ ), such a moment is easier to generate in the configuration of Fig. 1(a), when the $\mathrm{Cu}$ I spins can cant so that $\mathbf{M}_{\mathrm{I}} \perp \mathbf{M}_{\mathrm{I}}^{\dagger}$, than in that of Fig. 1(b), which would require $\mathbf{M}_{\mathrm{I}} \| \mathbf{M}_{\mathrm{I}}^{\dagger}$. This yields a fourfold symmetry, which prefers ordering of $\mathbf{M}_{\mathrm{II}}$ along (110), as indeed observed experi- mentally at low $H$. An additional fourfold anisotropy energy, of the form $\mathcal{H}_{4}=K \cos 4 \theta$, with $K>0$, which also prefers ordering along (110), has been shown to arise for the $\mathrm{Cu}$ I's in other cuprates, due to quantum fluctuations [12]. (It does not arise as a single ion term for $S=1 / 2$.) Indeed, our quantitative fits to the data require adding $\mathcal{H}_{4}$, with $K$ close to the value predicted in Ref. [12].

The anisotropic interactions in Eq. (1) could result from the usual dipole-dipole interactions. For the $\mathrm{NN}$ bonds, this yields $J^{\|}=-2 J^{\perp}=-2\left(g \mu_{B}\right)^{2} / r^{3} \approx-20 \mu \mathrm{eV}$, where $r=a / 2$ is the $\mathrm{Cu} \mathrm{I}-\mathrm{Cu} \mathrm{II}$ bond length, so that $J_{\text {pd }}<0$. However, similar anisotropic terms may also arise from direct or superexchange interactions involving spin-orbit and Coulomb exchange interactions, and these sometimes yield $J_{\mathrm{pd}}>0$, implying a flipping of $\mathbf{M}_{\mathrm{II}}$ in Fig. 1 [12]. Therefore, we call these terms pseudodipolar. A measurement of the relative directions of the spins would identify the sign of $J_{\mathrm{pd}}$.

Adding $\mathbf{H}$, Eq. (2) becomes $\mathcal{H}=-2 \mathbf{H} \cdot \mathbf{M}_{\mathrm{I}}-\mathbf{H}_{\mathrm{II}}$. $\mathbf{M}_{\text {II }}$, with $\mathbf{H}_{\text {II }}=\mathbf{H}-4 J_{\text {av }} \mathbf{M}_{\mathrm{I}}-4 J_{\mathrm{pd}} \hat{\Gamma} \mathbf{M}_{\mathrm{I}}^{\dagger}$. (The factor 2 is the number of $\mathrm{Cu}$ I's per planar unit cell, which contains one $\mathrm{Cu}$ II.) Assuming a linear response of the $\mathrm{Cu}$ II's, i.e., $\mathbf{M}_{\mathrm{II}} \approx \chi_{\mathrm{II}} \mathbf{H}_{\mathrm{II}}$, one finds a mean field energy per unit cell $E=E_{0}(I)-2 \mathbf{H} \cdot \mathbf{M}_{\mathrm{I}}-\chi_{\mathrm{II}}\left(\mathbf{H}_{\mathrm{II}}\right)^{2} / 2+\mathcal{H}_{4}$, where $E_{0}(I)$ contains the unperturbed energy of the Cu I's. For $T \ll T_{N, \mathrm{I}}$, it is sufficient to use a low temperature approximation, in which $E_{0}(I)=4 J_{0}\left[\mathbf{M}_{\mathrm{I}}^{2}-\left(\mathbf{M}_{\mathrm{I}}^{\dagger}\right)^{2}\right],\left(\mathbf{M}_{\mathrm{I}}\right)^{2}+$ $\left(\mathbf{M}_{\mathrm{I}}^{\dagger}\right)^{2}=\left(\mathbf{S}_{\mathrm{I}}\right)^{2} \equiv S^{2}$, and $\mathbf{M}_{\mathrm{I}} \perp \mathbf{M}_{\mathrm{I}}^{\dagger}$, implying that (without quantum corrections) $\chi_{\mathrm{I}, \perp}(T) \approx \chi_{\mathrm{I}, \perp}(0)=1 /\left(8 J_{0}\right)$ and $\chi_{\mathrm{I}, \|}=0$. Neglecting $J_{\mathrm{pd}}^{2}, J_{\mathrm{pd}} H$ in comparison with $J_{\mathrm{av}}^{2}$ and minimizing with respect to $M_{\mathrm{I}}$ gives $E(\theta)=-\left[\chi_{\mathrm{II}}+2 \tilde{\chi} \sin ^{2}(\theta-\alpha)\right] H^{2} / 2+M_{0} H[\cos (\theta+$ $\alpha)+y \sin 2 \theta \sin (\theta-\alpha)]-k \sin ^{2} 2 \theta+$ const, where $\tilde{\chi}=\left(1-2 J_{\mathrm{av}} \chi_{\mathrm{II}}\right)^{2} /\left[8\left(J_{0}-\chi_{\mathrm{II}} J_{\mathrm{av}}^{2}\right)\right] \approx \chi_{\mathrm{I}, \perp}(0), M_{0}=$ $4 J_{\mathrm{pd}} \chi_{\mathrm{II}} M_{\mathrm{I}}^{\dagger}, y=-4 J_{\mathrm{av}} \tilde{\chi} /\left(1-2 J_{\mathrm{av}} \chi_{\mathrm{II}}\right)$, and $k=2 K+$ $\left(M_{0} y / 2\right)^{2} / \tilde{\chi}$. Having solved $\partial E / \partial \theta=0$ for $\theta$, the measured moment is

$$
\begin{aligned}
M= & -\partial E / \partial H=\left[\chi_{\mathrm{II}}+2 \tilde{\chi} \sin ^{2}(\theta-\alpha)\right] H \\
& -M_{0}[\cos (\theta+\alpha)+y \sin 2 \theta \sin (\theta-\alpha)] .
\end{aligned}
$$

Equation (3) reproduces all of our experimental results. For $\alpha=\pi / 4$, the minimum occurs at $\theta=\pi / 4+$ $\operatorname{sgn}\left(J_{\mathrm{pd}}\right) \pi / 2$, leading to the structure shown in Fig. 1(a) and to $\chi^{(110)}=\left(\chi_{\mathrm{II}}+2 \tilde{\chi}\right)$ and $M_{S}^{(110)}=\left|M_{0}\right|(1+y)$. For very large $H$, the minimum obeys $\theta-\alpha \approx$ $\operatorname{sgn}\left(J_{\mathrm{pd}}\right) \pi / 2-M_{S}^{(110)} \cos 2 \alpha / 2 \tilde{\chi} H$, and hence $M=$ $\chi^{(110)} H+M_{S}^{(110)} \sin 2 \alpha+\mathcal{O}(1 / H)$, consistent with the parallel asymptotes in Fig. 3. In this limit, $\mathbf{M}_{\mathrm{I}}^{\dagger} \perp \mathbf{H}$.

For $\alpha=0$, the equation $\partial E / \partial \theta=0$ exhibits special behavior: $\sin \theta=0$ is always an extremum. When the quadratic equation $2 \tilde{\chi} H^{2}-\left|M_{0}\right|(1-4 y) H+8 k=0$ has two real and positive solutions $H_{c 1,2}$, then $\sin \theta=0$ is the only solution for $H_{c 1}<H<H_{c 2}$. This gives the structure of Fig. 1(b), and the straight $M(H)$ with $\chi^{\mathrm{b}}=\chi_{\mathrm{II}}$ and $M_{S}^{\mathrm{b}}=\left|M_{0}\right|$. For other values of $H$, the 
minimum has a nonzero $|\cos \theta|$, which starts at $\sqrt{2} / 2$ for $H=0$ [yielding the structure in Fig. 1(a)], increases towards 1 at $H=H_{c 1}$, where it remains up to $H_{c 2}$, corresponding to Fig. 1(b), and decreases towards 0 [i.e., Fig. 1(c)] as $H$ increases towards $\infty$ above $H_{c 2}$. This reproduces our data at $200 \mathrm{~K}$, and relates them to spin rotations between Figs. 1(a)-1(c). For all $\alpha \neq 0$, and also when the solutions $H_{c 1,2}$ do not exist (as happens at lower $T$ ), the solution $\sin \theta=0$ never applies, the sharp transitions disappear, and there is only one continuous solution for $\theta$.

Having extracted $M_{S}^{(110)}$ and $\chi^{(110)}$ from the data for $\alpha=\pi / 4$ (results given in Fig. 2), we next plot $m(H, \alpha)$ for other $\alpha$ 's. The function $m$ depends only on $\tilde{\chi} / M_{S}^{(110)}$, $y$, and $k / \tilde{\chi}$. All of our data for $70 \leq T \leq 120 \mathrm{~K}$ are consistent with Eq. (3), with the temperature independent parameters $\tilde{\chi}=(0.22 \pm 0.01) \times 10^{-6} \mathrm{~cm}^{3} / \mathrm{g}, y=$ $0.03 \pm 0.02$, and $K=(10 \pm 3) \times 10^{-7} \mathrm{meV}$ (full lines in Fig. 3). The error bars represent ranges of acceptable fits. The agreement with the data is excellent: $m(H, \alpha)$ turns out to be sensitive to $\alpha$ near 0 , and our "(100)" data are fit best with $\alpha \approx 0.2^{\circ}-0.4^{\circ}$, which is within the alignment error. From $y$ and $\tilde{\chi}$ we extract $J_{\mathrm{av}} \approx-(12 \pm$ 9) $\mathrm{meV}$. Using the quantum renormalization $\chi_{\mathrm{I}, \perp} \approx$ $0.53 / 8 J_{0}$ [17], $\tilde{\chi}$ gives $J_{0}=(130 \pm 40) \mathrm{meV}$, roughly the same as in other cuprates. $K$ is in rough agreement with the predictions of Ref. [12]. It has been too small to be measured directly in other cuprates. Using $M_{\mathrm{I}}^{\dagger} \approx 0.3$ [17], we finally find $\left|J_{\mathrm{pd}}\right|=(27 \pm 1) \mu \mathrm{eV}$, which is of the same order as the dipolar $J_{\mathrm{pd}} \approx-20 \mu \mathrm{eV}$. Our measured isotropic interaction $J_{\mathrm{av}}$ is much larger and FM, probably indicating superexchange and direct exchange. The graph for $200 \mathrm{~K}$ has somewhat different parameters: $\tilde{\chi}=(0.165 \pm 0.006) \times 10^{-6} \mathrm{~cm}^{3} / \mathrm{g}, y=0.008 \pm$ 0.004 , and $K=(5 \pm 1) \times 10^{-7} \mathrm{meV}$. At higher $T, \chi_{\mathrm{I}, \perp}$ should be replaced by $\chi_{\mathrm{I}, \perp}-\chi_{\mathrm{I}, \|}$, a quantity whose decrease to zero as $T$ increases towards $T_{N, \mathrm{I}}$ explains the observed decrease in $\tilde{\chi}$. The $T$ dependence of $y$ and $K$ is not yet understood. Unlike the parameters used at low $T$, the parameters at $200 \mathrm{~K}$ yield spin rotation transitions and reproduce the experimentally observed $H_{c 1}$ and $H_{c 2}$.

In addition to providing convincing evidence that the pseudodipolar interaction is the source of the ferromagnetism in 234, our experiments yield quantitative measurements of the previously unknown parameters $K, J_{\mathrm{II}}$, $J_{\mathrm{av}}$, and $J_{\mathrm{pd}}$. Results below $T_{N, \mathrm{II}}$ also confirm our theory [13]. It would be interesting to compare our values of $J_{\mathrm{av}}$ and $J_{\mathrm{pd}}$ with the $\mathrm{Cu}-\mathrm{Cu}$ interladder coupling $\left(J_{\mathrm{av}}\right.$ has been predicted to be FM there [10], but is thought to be AFM for the NN interaction in the chain system [9]). Since the isotropic interladder coupling is also frustrated, we expect it to be dominated by our $J_{\mathrm{pd}}$. It would also be interesting to understand the relevance of $J_{\text {II }}$ to the $\mathrm{NNN} \mathrm{Cu}-\mathrm{Cu}$ coupling in other lamellar cuprates and to study the consequences of our values of $J_{\mathrm{av}}, J_{\mathrm{pd}}$, and $J_{\mathrm{II}}$ for the NN and NNN interactions in the chains. Our results imply a large $\mathrm{NNN} / \mathrm{NN}$ ratio which could explain the mysterious gap observed there [9]. The sign of $J_{\mathrm{pd}}$ should determine if the chain spins prefer ordering parallel or perpendicular to the chains.

We acknowledge discussions with P. A. Lee, G. Shirane, and J. Stein. This work has been supported primarily by the MRSEC Program of the National Science Foundation under Award No. DMR 94-00334 at MIT, the U.S.-Israel Binational Science Foundation (at Tel Aviv, MIT, and Penn), the Israel Academy (at Tel Aviv) and NSF Grant No. DMR-95-20175 (at Penn).

[1] M. Greven, R. J. Birgeneau, Y. Endoh, M. A. Kastner, M. Matsuda, and G. Shirane, Z. Phys. B 96, 465 (1995).

[2] B. Keimer, A. Aharony, A. Auerbach, R. J. Birgeneau, A. Cassanho, Y. Endoh, R. W. Erwin, M. A. Kastner, and G. Shirane, Phys. Rev. B 45, 7430 (1992).

[3] B. Keimer, N. Belk, R. J. Birgeneau, A. Cassanho, C. Y. Chen, M. Greven, M. A. Kastner, A. Aharony, Y. Endoh, R. W. Erwin, and G. Shirane, Phys. Rev. B 46, 14034 (1992).

[4] S. Noro, T. Kouchi, H. Harada, T. Yamadaya, M. Tadokoro, and H. Suzuki, Mater. Sci. Eng. B 25, 167 (1994).

[5] H. Ohta, M. Sumikawa, M. Motokawa, S. Noro, and T. Yamadaya, J. Phys. Soc. Jpn. 64, 1759 (1995).

[6] K. Yamada, N. Suzuki, and J. Akimitsu, Physica (Amsterdam) 213\&214B, 191 (1995).

[7] B. Grande and H. Müller-Buschbaum, Z. Naturforsch. 31B, 405 (1976).

[8] M. Greven et al. (unpublished).

[9] M. Matsuda and K. Katsumata, Phys. Rev. B 53, 12201 (1996).

[10] E. Dagotto and T. M. Rice, Science 271, 618 (1996).

[11] T. Thio, T. R. Thurston, N. W. Preyer, P. J. Picone, M. A. Kastner, H. P. Jenssen, D. R. Gabbe, C. Y. Chen, R. J. Birgeneau, and A. Aharony, Phys. Rev. B 38, 905 (1988).

[12] T. Yildirim, A. B. Harris, A. Aharony, and O. EntinWohlman, Phys. Rev. B 52, 10239 (1995).

[13] F. C. Chou et al. (unpublished).

[14] T. Thio and A. Aharony, Phys. Rev. Lett. 73, 894 (1994).

[15] P. Selwood, Magnetochemistry (Interscience, New York, 1956), p. 78.

[16] D. C. Johnston, Phys. Rev. Lett. 62, 957 (1989).

[17] M. Makivic and H.-Q. Ding, Phys. Rev. B 43, 3562 (1991); M. Greven, Ph.D. thesis, M.I.T., 1995.

[18] In the theory we use dimensionless moments $S, M$, and measure the various $J$ 's, $H$, and $1 / \chi$ in ergs. To translate into the experimental units of emu/g and $\mathrm{cm}^{3} / \mathrm{g}$, multiply $M$ by $g \mu_{B} / m_{u c}=22.4 \mathrm{emu} / \mathrm{g}$, where $g=2$ and $m_{u c}=500 \mathrm{~g} / N_{A}=83 \times 10^{-23} \mathrm{~g}$, and $\chi$ by $\left(g \mu_{B}\right)^{2} / m_{u c}=4.16 \times 10^{-19} \mathrm{erg} \mathrm{cm}^{3} / \mathrm{g}$. Using $g=2.2$ will modify some of the parameters slightly.

[19] In fact, a low $T$ state with finite $\mathbf{M}_{\mathrm{I}} \| \mathbf{M}_{\mathrm{II}} \perp \mathbf{M}_{\mathrm{I}}^{\dagger}$ would be generated by $J_{\mathrm{av}}$ alone even when $J_{\mathrm{pd}}=0$. However, this requires $J_{\mathrm{av}}^{2} \chi_{\mathrm{II}}>J_{0}$, which is not obeyed here. [E. F. Shender, Sov. Phys. JETP 56, 178 (1982); J. Stein, A. Aharony, O. Entin-Wohlman, and A. B. Harris (unpublished)]. 